\title{
Short-term effect of a developed warming moist chamber goggle for video display terminal-associated dry eye
}

\author{
Yueping Ren, Jie Chen, Qinxiang Zheng and Wei Chen*
}

\begin{abstract}
Background: Video display terminal (VDT)-associated dry eye (DE) patients are the rising group worldwide, and moisture goggles are the preferable treatment since they are capable of improving tear film stability and DE discomfort. The current study aims to evaluate the short-term efficacy and safety of the developed warming moist chamber goggles (WMCGs) for VDT-associated DE patients.

Methods: In this prospective self-control study, 22 DE patients (22 eyes) working with VDTs over $4 \mathrm{~h}$ daily were enrolled and instructed to wear WMCGs for 15 min. Sodium hyaluronate (SH, 0.1\%) eyedrops were applied as a control on another day on these same patients, however 4 subjects denied the eyedrop application. The symptomatology visual analog scale (VAS) score, tear meniscus height (TMH), noninvasive tear film break-up time (NI-BUT), tear film lipid layer thickness (LLT), and bulbar conjunctival redness were assessed with Keratograph $5 \mathrm{M}$ at baseline, 5, 30 and 60 min after treatment. The WMCGs wearing comfort was also evaluated.
\end{abstract}

Results: The ocular discomfort evaluated by VAS decreased in the WMCGs group throughout 60 min $(P<0.001)$, better than the control group levels $(P \leq 0.015)$. TMH, NI-BUT (including the first BUT and average BUT) increased than baseline level accross 60 min in the WMCG group $(P \leq 0.012)$, while those in the control group only showed temporary improvements in 5 min. LLT also increased obviously after WMCGs wear, while the change in the control group was nearly innoticeable. No adverse responses were detected.

Conclusions: Temporary use of the WMCGs is able to relieve ocular discomfort, and improves tear film stability in DE patients for at least $1 \mathrm{~h}$, making it a promising alternative to other treatments.

Keywords: Dry eye, Video display terminal, Warming moist chamber goggles, Tear film

\section{Background}

In modern society, some external and occupational environments can be risk factors for the development of dry eye (DE), including low relative humidity, high wind velocity, air conditioning, and long time working with video display terminals (VDTs) [1-4]. Excessive tear evaporation and tear film instablity can be caused by these conditions and induce dry eye symptoms, such as eye dryness, burning, gritty, itchy, scratchiness, soreness, and blurred vision [5]. Nearly $13.1 \%$ of the middle-aged Japanese VDT users experience dry eye symptoms in a large sample investigation [6]. Excessive VDT use results

* Correspondence: weichenmd@hotmail.com

School of Ophthalmology and Optometry, Wenzhou Medical University, 270 Xueyuan West Road, Wenzhou, Zhejiang 325027, People's Republic of China in decreased blink rates, which then delay the coating of the meibomian gland-sourced lipid layer over the aqueous layer, and consequently increase tear evaporation rates [7]. The instable supply of the lipid layer plays a vital role in the development of DE in VDT users. The VDT-associated dry eye patients is an increasing DE group in the modern world, suffering from eye discomfort or even mental fatigue and headache [8].

To increase the lipid layer thickness (LLT) is the suggested way to decrease tear evaporation and stablize the tear film in DE patients with lipid layer malfunction $[9,10]$. Different methods have been used in clinic for the purpose of thickening lipid layer including manual massage, heat compress, various types of goggles and the rising therapy of intense pulsed light (IPL) $[11,12]$. The 
traditional heat compress is usually inconvenient with poor patient compliance, while the IPL therapy is currently cost-prohibitive and still need more investigations. Moisture goggles or eyelid warming devices are reported to be capable of improving tear film stability, lipid layer thickness, as well as dry eye symptoms and ocular comfort $[9,13-15]$. Besides, healthy subjects also benifit from these devices since 10 mins treatment of a eyelidwarming device could provided more effective warming and ocular surface comfort than warm compresses [16]. However, the previous studies usually focused on the effects of the devices on meibomian gland dysfunction (MGD) patients, and little results were available for VDT-associated DE. Thus in the current study, we develped a new warm moist chamber goggles (WMCGs) to deliver heat to the periocular area and increase the humidity by attaching moist sponges in a sealed periocular chamber, and evaluated the short term effects of this device on VDT-associated DE subjects, including DE discomfort relief, the changes of noninvasive tear breakup time (NI-BUT), tear meniscus height (TMH) and LLT measured by Keratograph $5 \mathrm{M}$, compared with the results of the $0.1 \%$ sodium hyaluronate $(\mathrm{SH})$ eyedrops.

\section{Methods}

\section{Subjects}

In this prospective self-control study, a total of $22 \mathrm{DE}$ patients (22 eyes) aged at $26.5 \pm 4.85$ years were enrolled in the Ocular Surface Center of the Eye Hospital of Wenzhou Medical University. The use of the chamber goggles is currently a part of standard care for DE patients, and the whole procedure of the study was approved by the Institutional Review Board of Wenzhou Medical University and adhered to the tenets of the Declaration of Helsinki.

Written informed consent was obtained from all subjects before participating in the study. The inclusion criteria consisted of DE symptoms persisting for at least 3 months with two or more than two kinds of symptoms such as irritation, eye desiccation, burning sensation, redness, reduced visual acuity, ocular pain, double vision, eyestrain, or tiredness; working in offices with VDTs more than $4 \mathrm{~h}$ everyday, since long time of VDT work (more than $4 \mathrm{~h}$ daily) was observed to be associated with a high incidence of DE disease [6, 17]; an OSDI (ocular surface disease index) over 13 [18, 19]; a fluorescein TBUT of $<5 \mathrm{~s}$ or a Schirmer I test score of $\leq$ $5 \mathrm{~mm} / 5 \mathrm{~min}$; no use of topical anti-inflammatory drugs (i.e. 0.05\% cyclosporine A or steroids) except for artificial tears that has been stopped for at least 1 week. Patients with a history of ocular surgeries, any ocular diseases, contact lens use, any systemic diseases or medication, or perimenopausal, pregnant and lactating women were excluded. For every enrolled patient, both eyes received $0.1 \% \mathrm{SH}$ eyedrops and/or WMCGs wear, however only the eye with smaller
TBUT, or smaller Schirmer I test score if the TBUT is the same in both eyes, or the right eye if both TBUT and Schirmer I test score are the same in both eyes, was selected for statistical analysis.

\section{Study design}

A same doctor conducted the enrollment tests of the DE patients on the first day. They were asked to return to the study center to apply $0.1 \% \mathrm{SH}$ eyedrops (Santen, Osaka, Japan) in both eyes for one time at 9 AM on the second day, and wear a pair of WMCGs for 15 min from 9 AM on the third day. The ambient temperature and humidity in the examination room were monitored and maintained at a range from 23 to $24{ }^{\circ} \mathrm{C}$ and 30 to $40 \%$ respectively, and the patients need to stay in the room for $20 \mathrm{~min}$ before treatments. Clinical outcome measures and subjective effects were assessed in the both eyes at baseline, 5, 30 and $60 \mathrm{~min}$ after eyedrop instillation or WMCGs wearing, including the visual acuity, the intraocular pressure (IOP), TMH, NI-BUT, tear film lipid layer, and bulbar conjunctival redness with Keratograph $5 \mathrm{M}$ (K5M; Oculus Optikgeräte $\mathrm{GmbH}$, Wetzlar, Germany), as well as OSDI scores. The safety and wearing comfort were also evaluated for the treatment with the WMCGs. The comparisons of the subjective and objective therapeutic effects of the two treatments were made breadthwise and lengthwise. The patients were asked to get up before 7 AM to minimize the influence of longtime eye closure on tear film [20], and avoid eye makeup and swimming recently.

\section{Subjective symptoms evaluation}

DE symptoms were evaluated according to the OSDI questionnaire by DEWS [21, 22]. Visual Analog Scale (VAS) were used to assess the degree of subjective discomfort [23]. Subjects were required to grade ocular discomfort from the baseline on a 0 to 10 scale, where 0 was no discomfort and 10 was the most discomfortable. Higher scores on the visual analog scale referred to a greater feeling of discomfort in the eyes in this study. Subjects were also inquired about their wearing comfort after WMCGs wear.

\section{Tear meniscus height}

Objective tear function parameters were measured with the Keratograph $5 \mathrm{M}$ in a darkened room. Lower tear film meniscus images were captured with the Keratograph $5 \mathrm{M}$ equipped with a modified tear film scan software. TMH was measured with an integrated ruler on the vertical line through the pupil center.

\section{Noninvasive tear film break-up time}

The Keratograph $5 \mathrm{M}$ automatic infrared illumination was deactivated, and the Placido rings were projected onto the cornea. After adjusting the focus, subjects were 
required to blink twice and to keep their eyes open for as long as possible during the NI-BUT measurements. Tear film topographic data were continuously recorded and discontinued at the next blink. In this study, first break-up time (FBUT) was defined as the time in seconds between the last complete blink before detection and the first distortion in the individual ring. The average break-up time (ABUT) is the mean time of the break-up timepoints during the whole detecting period.

\section{Corneal fluorescein staining}

Corneal fluorescein staining (CFS) was evaluated by instilling $0.5 \mathrm{ml}$ of $5 \%$ fluorescein solution into the inferior conjunctival sac using a micropipette. The cornea was examined using slit-lamp microscopy in cobalt blue light $3 \mathrm{~min}$ after fluorescein instillation. The staining in five different regions was assessed according to a standardized grading system ranging from 0 to 4 [24]. The total scores were used as an index of epithelial integrity.

\section{Lipid layer evaluation}

The white interference light was projected onto the tear film. The colors and structures of lipid layer were observed and video recorded in real time between multiple blinks. During the video recording, subjects were instructed to blink naturally. Analysis of the lipid layer was achieved by evaluating color interference patterns in zones of specular reflection at the air/lipid layer. If colors and structures are visible, the lipid layer is thick. If there are neither colors nor structures visible, the lipid layer is very thin.

\section{Bulbar Redness index (BRI)}

Nasal and temporal conjunctival bulbar areas were analyzed independently by the Keratograph $5 \mathrm{M}$. Subjects were instructed to open their eyes as widely as possible during the examination. Redness values were graded automatically ranging from 0 to 4 by the system. The final score was the average of the captured three values.

The WMCGs used in our study is a pair of goggles designed to provide moisture and suitable heat to the periocular region (Fig. 1). The heat is produced by the installed heat elements. The WMCGs need preheating for $6 \mathrm{~min}$, then moisture the attached sponges with $1 \mathrm{ml}$ $0.9 \%$ sterile saline. Wear the goggle for 15 min when the light turned from red to blink. The local temperature maintained at $40{ }^{\circ} \mathrm{C}$, and the treatment would stop when the light turned off. And the subjects were inquired about their satisfaction with the WMCGs wearing, which was reported as very comfortable, comfortable, or uncomfortable.

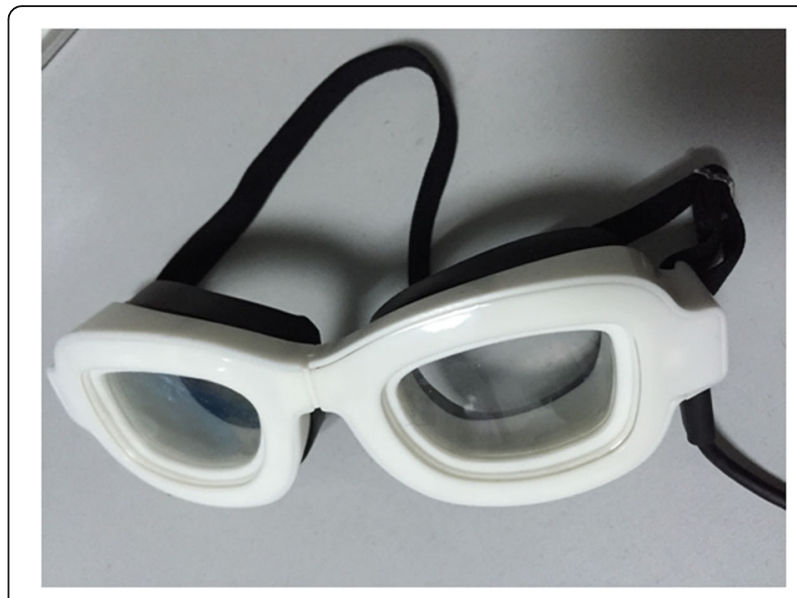

Fig. 1 The photo of the new moist chamber goggle

\section{Statistical analysis}

Statistical analysis was performed using the SPSS software version 20.0 (SPSS Inc., Chicago, IL). Comparisons of the subjective and objective parameters between the two methods of treatment at baseline, $5 \mathrm{~min}, 30 \mathrm{~min}$ and $60 \mathrm{~min}$ apply the independent $\mathrm{t}$ test if the data is of the normal distribution, or otherwise the Mann-Whitney $\mathrm{U}$ rank test. Comparisons of the data between each time point $(15,30$, and $60 \mathrm{~min})$ and baseline were conducted using the paired $t$ test if the data is of the normal distribution, or otherwise the Wilcoxon signed rank test with the Bonferroni correction. Two-tailed $P<0.05$ were considered to indicate a statistically significant difference.

\section{Results}

In this prospective self-control clinical study, 22 VDT users with dry eye in both eyes (14 women and 8 men; mean age, $26.5 \pm 4.85$ years) were recruited to evaluate the effect of WMCGs on tear functions, compared with those of once use of $0.1 \% \mathrm{SH}$ eyedrops. However 4 subjects ( 2 men and 2 women) denied to apply $0.1 \% \mathrm{SH}$ eyedrops and only finished the tests of WMCGs, making the control group 4 cases less than the WMCGs group. Table 1 shows the comparison of clinical characteristics of the involved subjects at baseline between the WMCGs group and the control $0.1 \% \mathrm{SH}$ group. No significant differences were found in age, sex distribution, the time of VDT use, OSDI score, or the tear function parameters between the two groups $(P \geq 0.244)$.

\section{Subjective VAS score and wearing satisfaction}

Relief in ocular discomfort, which was evaluated by VAS, was observed after $5 \mathrm{~min}$ in the both groups. VAS in the WMCGs group decreased from $5.25 \pm 2.00$ at baseline to $1.68 \pm 1.79$ at $5 \mathrm{~min}$ after goggles wear $(P<0.001)$, and it kept significantly reduced throughout $60 \mathrm{~min}(P<0.001)$; while in the control group, the improvement existed at 
Table 1 The pretreatment clinical characteristics of the subjects in the WMCGs and the $0.1 \%$ SH groups

\begin{tabular}{llll}
\hline & WMCG group $(N=22)$ & $0.1 \%$ SH group $(N=18)$ & \\
& Median $(25 \%, 75 \%) /$ & Median $(25 \%, 75 \%) /$ & $P$ \\
& Mean \pm SD & Mean \pm SD & \\
\hline Age (Year) & $26.5(25,28.25)$ & $26(25,27.25)$ & 0.510 \\
Gender (M:F) & $8: 14$ & $6: 12$ & 0.968 \\
VDT (h) & $9.41 \pm 1.87$ & $9.79 \pm 2.01$ & 0.570 \\
FBUT (s) & $3.49 \pm 0.97$ & $3.52 \pm 1.22$ & 0.665 \\
ABUT (s) & $4.86 \pm 1.34$ & $4.92 \pm 1.69$ & 0.936 \\
CFS & $1(0,3.25)$ & $0(0,2)$ & 0.244 \\
SIt (mm) & $16.49 \pm 10.98$ & $15.63 \pm 11.69$ & 0.824 \\
IOP (mmHg) & $14.51 \pm 2.38$ & $15.00 \pm 2.18$ & 0.434 \\
OSDI & $37.49 \pm 15.24$ & $36.93 \pm 16.69$ & 0.918 \\
\hline
\end{tabular}

WMCGs warming moist chamber goggles, $S H$ sodium hyaluronate, VDT video display terminals, FBUT first break-up time, ABUT average break-up time, CFS corneal fluorescein staining, S/t Shirmer I test, IOP intraocular pressure, OSDI ocular surface disease index
5 min (from baseline $5.07 \pm 1.93$ to $4.26 \pm 2.02, P=0.006$ ) and $30 \mathrm{~min}(4.51 \pm 1.83, P=0.017)$ after $0.1 \% \mathrm{SH}$ application, but not at $60 \mathrm{~min}(P=0.058)$. The VAS was much lower in the WMCGs group than that in the control group across all the 3 timepoints $(P \leq 0.015)$ (Table 2, Fig. 2a). Wearing comfort of the WMCGs was reported as $27.27 \%(6 / 22)$ very comfortable, $68.18 \%(15 / 22)$ comfortable, and $4.55 \%(1 / 22)$ uncomfortable.

\section{Objective tear film functions}

TMH increased significantly throughout the $60 \mathrm{~min}$ in the WMCGs group, which raised from $0.18 \pm 0.05$ at baseline to $0.20 \pm 0.06$ at $5 \mathrm{~min}(P=0.004)$, and kept elevated at $30 \mathrm{~min}(P=0.001)$ and $60 \mathrm{~min}(P=0.012)$. However in the control group, the improvement only existed at $5 \mathrm{~min}$ after $0.1 \% \mathrm{SH}$ application $(P=0.001)$. And no significant changes were observed in TMH across all timepoints between the two groups $(P \geq 0.341)$ (Table 2, Fig. 2b).

FBUT in the WMCGs group increased distinctively from $3.49 \pm 0.97$ at baseline to $10.22 \pm 7.21$ after $5 \mathrm{~min}$ $(P<0.001)$, and the improvement kept significant throughout $60 \mathrm{~min}(P=0.001)$. In the $0.1 \% \mathrm{SH}$ group,

Table 2 The changes of VAS, TMH, FBUT and ABUT after treatment in the WMCGs and the $0.1 \%$ SH groups

\begin{tabular}{|c|c|c|c|c|c|}
\hline & \multicolumn{2}{|c|}{ WMCG group $(N=22)$} & \multicolumn{2}{|c|}{$0.1 \% \mathrm{SH}$ group $(N=18)$} & \multirow[b]{2}{*}{$P^{* *}$} \\
\hline & Mean \pm SD & $P^{*}$ & Mean \pm SD & $P^{*}$ & \\
\hline \multicolumn{6}{|l|}{ VAS } \\
\hline Pre-treatment & $5.25 \pm 2.00$ & N/A & $5.07 \pm 1.98$ & N/A & 0.800 \\
\hline 5 min post-treatment & $1.68 \pm 1.79$ & $<0.001$ & $4.26 \pm 2.02$ & 0.006 & $<0.001$ \\
\hline 30 min post-treatment & $2.80 \pm 1.96$ & $<0.001$ & $4.51 \pm 1.83$ & 0.017 & 0.015 \\
\hline 60 min post-treatment & $2.76 \pm 1.84^{\mathrm{a}}$ & $<0.001$ & $4.60 \pm 1.69$ & 0.058 & 0.005 \\
\hline \multicolumn{6}{|l|}{$\mathrm{TMH}$} \\
\hline Pre-treatment & $0.18 \pm 0.05$ & N/A & $0.18 \pm 0.05$ & N/A & 0.701 \\
\hline 5 min post-treatment & $0.20 \pm 0.06$ & 0.004 & $0.21 \pm 0.05$ & 0.001 & 0.642 \\
\hline 30 min post-treatment & $0.21 \pm 0.07$ & 0.001 & $0.19 \pm 0.05$ & 0.275 & 0.341 \\
\hline 60 min post-treatment & $0.20 \pm 0.06$ & 0.012 & $0.18 \pm 0.05$ & 0.927 & 0.524 \\
\hline \multicolumn{6}{|l|}{ FBUT } \\
\hline Pre-treatment & $3.49 \pm 0.97$ & N/A & $3.52 \pm 1.22$ & N/A & 0.936 \\
\hline 5 min post-treatment & $10.20 \pm 7.21$ & $<0.001$ & $5.10 \pm 1.76$ & 0.011 & 0.004 \\
\hline 30 min post-treatment & $6.06 \pm 2.85^{\mathrm{a}}$ & 0.001 & $5.74 \pm 3.93$ & 0.077 & 0.783 \\
\hline 60 min post-treatment & $6.34 \pm 3.69^{\mathrm{a}}$ & 0.001 & $3.87 \pm 1.26$ & 0.463 & 0.007 \\
\hline \multicolumn{6}{|l|}{ ABUT } \\
\hline Pre-treatment & $4.86 \pm 1.34$ & N/A & $4.92 \pm 1.69$ & N/A & 0.890 \\
\hline 5 min post-treatment & $14.29 \pm 6.38$ & $<0.001$ & $6.86 \pm 2.28$ & 0.004 & $<0.001$ \\
\hline 30 min post-treatment & $8.83 \pm 4.31^{\mathrm{a}}$ & 0.002 & $6.98 \pm 4.29$ & 0.069 & 0.228 \\
\hline 60 min post-treatment & $8.05 \pm 3.89^{\mathrm{a}}$ & 0.001 & $5.22 \pm 1.60$ & 0.566 & 0.015 \\
\hline
\end{tabular}

$P<0.05$ was regarded as significant difference

VAS visual analog scale, TMH tear meniscus height, FBUT first break-up time, ABUT average break-up time, WMCGs warming moist chamber goggles, SH sodium hyaluronate *Self-comparison (post-treatment values compared with baseline level) by paired t test with Bonferroni correction

**Comparison between the two groups at the same timepoint by independent $t$ test

${ }^{a}$ Comparison between the value at 30 or $60 \mathrm{~min}$ and at $5 \mathrm{~min}$ after treatment 

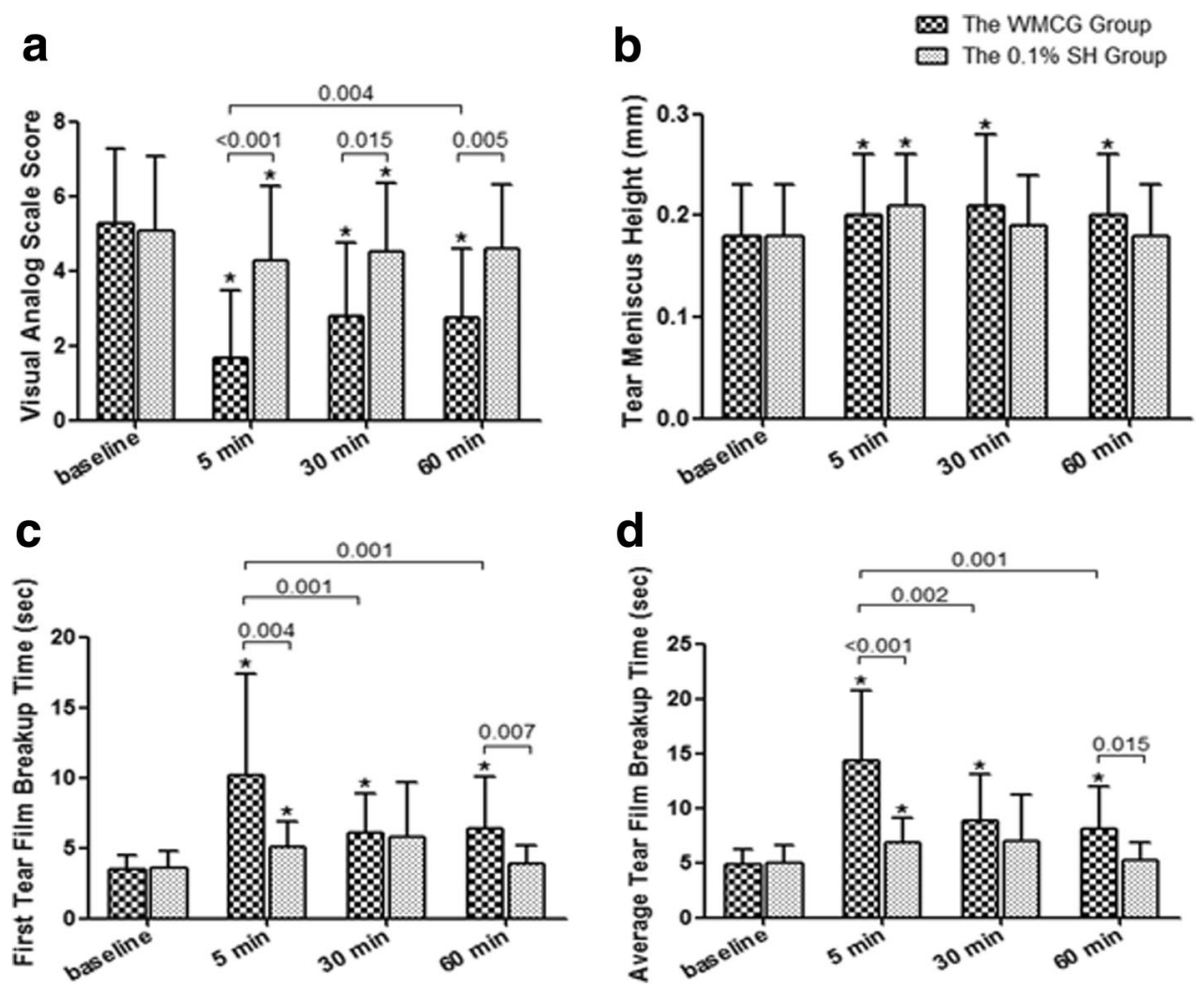

Fig. 2 The changes of VAS, TMH, FBUT and ABUT after treatment in the WMCGs and the $0.1 \%$ SH groups. The VAS (a), TMH (b), FBUT (c) and ABUT (d) improved significantly across 60 min after wearing goggles for 15 mins ( $P \leq 0.012$ ); however the improvement only existed for these parameters at 5 min after $0.1 \% \mathrm{SH}$ application in the control group $(P \leq 0.011)$, as well as for VAS at $30 \mathrm{~min}(P=0.017)$. The difference bwtween the two groups was significant for VAS across $60 \mathrm{~min}(P \leq 0.015)$, for FBUT and ABUT at $5 \mathrm{~min}$ and $60 \mathrm{~min}(P \leq 0.015)$, but bot for TMH $(P \geq 0.341)$. * Self-comparison (posttreatment values compared with baseline level) by paired t test with Bonferroni correction $(P<0.05)$

the increase is significant at $5 \mathrm{~min}(P=0.011)$ but not in later timepoints $(P \geq 0.077)$. The difference between the WMCGs and the control groups is significant at $5 \mathrm{~min}$ and $60 \mathrm{~min}(P \leq 0.007)$, but not at $30 \mathrm{~min}$ (Table 2, Fig. 2c). Similarly, ABUT in the WMCGs group also increased from baseline $4.86 \pm 1.34$ to $14.29 \pm 6.38$ after 5 min with a great raise $(P<0.001)$, which remained significant across $60 \mathrm{~min}(P \leq 0.002)$. In comparison, ABUT also increased in the control group 5 min after $0.1 \% \mathrm{SH}$ installation $(P=0.004)$, but the effect did not keep for $30 \mathrm{~min}(P \geq 0.069)$. And significant difference was observed between the two groups at $5 \mathrm{~min}$ as well as at 60 min $(P \leq 0.015)$ (Table 2, Fig. 2d).

Obvious improvements in LLT after wearing WMCGs were noted (Fig. 3). The lipid layer structure was very thin with pretty grey color, which was hardly visible at baseline. After $5 \mathrm{~min}$ of finishing WMCGs wear, the LLT became much thicker with brown-blue interference patterns in 13 (59.09\%) eyes, which kept for $30 \mathrm{~min}$ and decreased gradually, however it was still thicker than the baseline level. In another 8 (36.36\%) eyes, the lipid layer became thicker with brown color across $30 \mathrm{~min}$, but it returned to the baseline level after $60 \mathrm{~min}$. And no big difference in LLT was observed in the other 1 (4.55\%) eye. However the effect in the control $0.1 \%$ SH group (18 eyes) was not comparable, since only 3 (16.67\%) eyes has mild increase in LLT after $5 \mathrm{~min}$, which turned unnoticeable after $30 \mathrm{~min}$, and no changes was found in the other 15 (83.33\%) eyes.

No ocular side effects were reported with the use of WMCGs or $0.1 \% \mathrm{SH}$ eyedrops. There were no differences in the visual acurity $(P=0.957)$ and IOP $(P=$ $0.531)$ before and after WMCGs wear. BRI also did not show significant difference at the 3 timepoints after treatment both in the WMCGs $(P \geq 0.151)$ and the control group $(P \geq 0.185)$.

\section{Discussion}

In the current study, we used the developed warming moist chamber goggles to create a comfortable periocular condition in a sealed chamber, in which the temperature $\left(40{ }^{\circ} \mathrm{C}\right)$ and humidity are well controlled. Constant and efficient eyelid warming melts the meibum oil and increases the secretion of meibum oil, which consequently improve the function tear film lipid layer. And the addition of moist sponges attached to the goggles produces steady water evaporation, which increases the periocular chamber humidity and provides a moist 

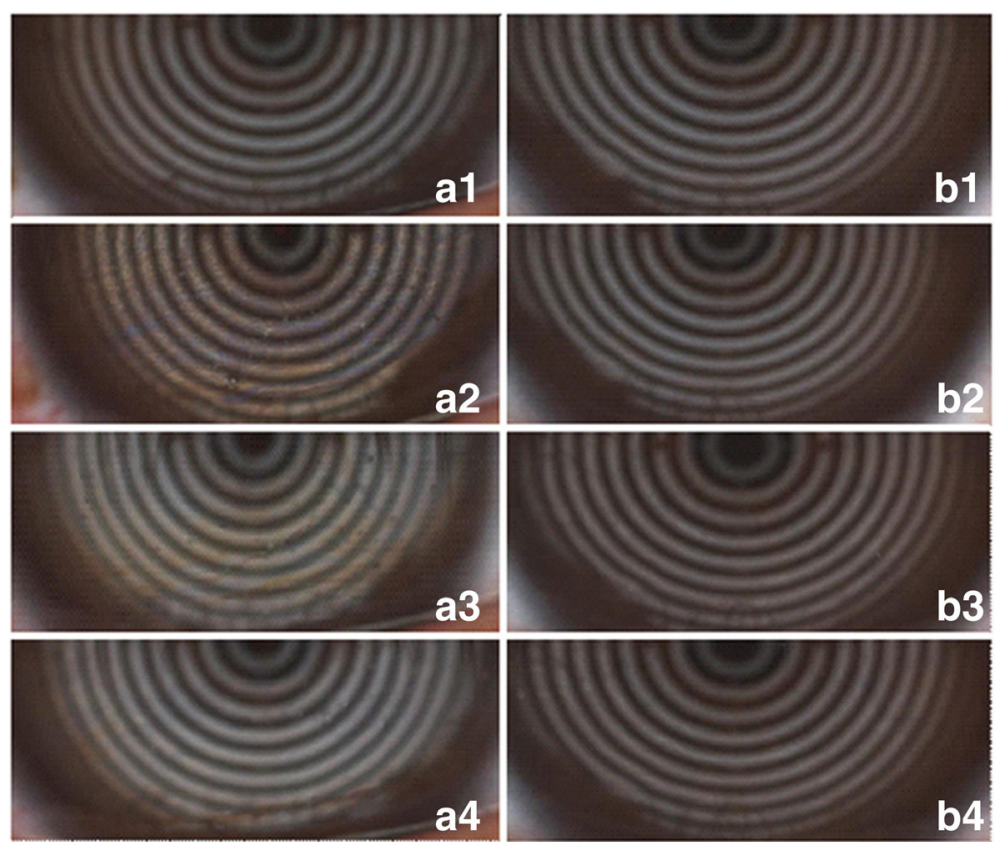

Fig. 3 The representative pictures showing the improvements in lipid layer thickness after WMCGs wear. The lipid structure was very thin with pretty grey color and hardly visible at baseline (a1, b1). The LLT became much thicker with brown-blue interference patterns after 5 min of finishing WMCGs wear (a2), and the effect kept similar after $30 \mathrm{~min}$ (a3), and decreased but still thicker than the baseline level after 60 min (a4). However the effect in the control group was nearly unnoticeable accross 60 min (b2, b3, and b4)

and warm ocular environment. Various types of eyelid warming or moisture devices have been shown to improve dry eye symptoms, ocular comfort, tear stability, and thickness of the tear film lipid layer in DE patients [9, 13-15]. And our WMCGs appear to have these positive effects as stated above.

Our results showed that ocular discomfort was significantly decreased in DE subjects after 15 min of WMCGs wear. The improvement in VAS lasted at least $60 \mathrm{~min}$ in the WMCGs group and the symptom relief was much better than the control group, indicating that the WMCGs is a preferable device for DE patients. In Korb er al.'s study, swim goggles improved DE ocular discomfort, however the symptoms returned in $91.9 \%$ of the patients $15 \mathrm{~min}$ after the goggles were removed [15]. Our WMCGs provide a longer period on DE symptom relief after goggle removal because their swim goggles don't have the stable warming and moisture functions. However, the effect of mid/long term and mutiple use of the WMCGs needs to be investegated, to help to guide the standard use of the goggles.

The results of tear film parameters were in accord with the subjective symptom melioration. The advanced placido topograph Keratograph $5 \mathrm{M}$, is a recommended device to measure NI-BUT and TMH $[25,26]$, and it is also objective and reliable to score bulbar redness [27]. It has been a widely used tool to evaluate tear film and DE diagnosis in clinic [28]. In this study, we found that the TMH, NI-BUT and ABUT increased significantly 5 min after the application of WMCGs wear or the 0.1\% SH eyedrops. However, the WMCGs wear has prolonged effect on tear film since these perameters were still better than the baseline level across $60 \mathrm{~min}$ while those in the control group failed to exert such effect. Bilkhu et al. reported that after a treatment period of an EyeBag eyelid warming device, significant improvements could be observed in TMH, BUT, LLT, ocular surface staining, and conjunctival hyperaemia in tested eyes compared with the contralateral control eye [29]. Simiar results has been demonstrated in other types of eyelid warming devices $[9,30]$, however these studies mainly focused on MGD patients while our results were obtained from VDT-associated DE patients, which is rarely discussed before.

Besides, the lipid layer thickness was significantly increased after WMCGs wear for $15 \mathrm{~min}$ in $95 \%$ subjects, and the improvement can last at least for $60 \mathrm{~min}$. But the application of $0.1 \% \mathrm{SH}$ eyedrops produced unnoticeable changes for lipid layer thickening. Tsubota \& Nakamori demonstrated that the VDT use could induce a decreased frequency of blinking and an increased rate of tear evaporation, both of which contribute to DE development [31]. So the improvement of lipid layer thickness and stability is the core route to relieve or even get rid of DE symptoms. Tsubota's team recently displayed that the use of a moist cool air device (MCAD) was able 
to improve BUT and tear evaporation rate, but not the lipid layer stability and corneal staining scores for VDT workers [32]. The core difference between their MCAD and our WMCGs is the control of the temperature, since they cooled the periocular area while we warmed this area to a steady temperature of $40{ }^{\circ} \mathrm{C}$, which increases the lipid release and the lipid layer thickness, and subsequently decrease the tear evaporation. So our WMCGs is a better choice for VDT-associated DE patients.

The safety and wearing comfort have been approved by the subjects, and no adverse events were reported during this study. Our WMCGs deliver heat through a electrocircuit which is insulated in the goggle frame, and the maximal power of the electrocircuit can only reach $40{ }^{\circ} \mathrm{C}$, so the safety of the WMCG is well guaranteed. This study aims to show the short-term effect of once use of the WMCGs in the VDT-associated DE patients, while the long-term results with repeated use of the goggles would be provided in our further studies, as well as in the other types of DE patients like the MGD or Sjögren syndrome patients.

\section{Conclusions}

This study demonstrates that the short-term use of the developed WMCGs is able to improve ocular comfort, and increase TMH, NI-BUT and LLT in VDT-associated $\mathrm{DE}$ patients. It is a better alternative for $\mathrm{DE}$ patients who refuse to use multiple eyedrops or who have unfavorable effects of eyedrops. The environmentally friendly nature of the WMCGs would also be highly evaluated since it is resource-saving and can be used repeatedly for a long period.

\section{Abbreviations}

ABUT: Average break-up time; BRI: Bulbar Redness index; DE: Dry eye; FBUT: First break-up time; IOP: Intraocular pressure; IPL: Intense pulsed light; LLT: Lipid layer thickness; MCAD: Moist cool air device; MGD: Meibomian gland dysfunction; NI-BUT: Noninvasive tear film break-up time; OSDI: Ocular surface disease index; TMH: Tear meniscus height; VAS: Visual analog scale; VDT: Video display terminal; WMCGs: Warming moist chamber goggles

\section{Acknowledgements}

Not applicable.

\section{Funding}

This study was funded by research grants from Wenzhou Science and Technology Program (2016Y0681) and grants from Zhengjiang Medical Science and Technology Project (2017KY489)

\section{Availability of data and materials}

The datasets used and analysed during the current study are available from the corresponding author on reasonable request.

\section{Authors' contributions}

YR and WC analyzed and interpreted the patient data, and write the manuscript. JC performed the examination of the patients and collect the data. QZ is a contributor in analyzing the data. All authors read and approved the final manuscript.

\section{Ethics approval and consent to participate}

The whole procedure of the study was approved by the Institutional Ethics Board of Wenzhou Medical University and adhered to the tenets of the Declaration of Helsinki. Written informed consent was obtained from all subjects before participating in the study.

Consent for publication

Not Applicable.

\section{Competing interests}

The authors declare that they have no competing interests.

\section{Publisher's Note}

Springer Nature remains neutral with regard to jurisdictional claims in published maps and institutional affiliations.

Received: 24 November 2017 Accepted: 30 January 2018

Published online: 07 February 2018

\section{References}

1. Schlote T, Kadner G, Freudenthaler N. Marked reduction and distinct patterns of eye blinking in patients with moderately dry eyes during video display terminal use. Graefes Arch Clin Exp Ophthalmol. 2004;242:306-12.

2. Backman H, Haghighat F. Indoor-air quality and ocular discomfort. J Am Optom Assoc. 1999;70:309-16.

3. Abelson MB, Ousler GW 3rd, Nally LA, Welch D, Krenzer K. Alternative reference values for tear film break up time in normal and dry eye populations. Adv Exp Med Biol. 2002:506:1121-5.

4. Ousler GW 3rd, Abelson MB, Nally LA, Welch D, Casavant JS. Evaluation of the time to "natural compensation" in normal and dry eye subject populations during exposure to a controlled adverse environment. Adv Exp Med Biol. 2002;506:1057-63.

5. Reijula K, Sundman-Digert C. Assessment of indoor air problems at work with a questionnaire. Occup Environ Med. 2004;61:33-8.

6. Uchino M, Schaumberg DA, Dogru M, Uchino Y, Fukagawa K, Shimmura S, et al. Prevalence of dry eye disease among Japanese visual display terminal users. Ophthalmology. 2008;115:1982-8.

7. Yaginuma $Y$, Yamada $H$, Nagai $H$. Study of the relationship between lacrimation and blink in VDT work. Ergonomics. 1990;33:799-809.

8. Wolkoff P, Nojgaard JK, Troiano P, Piccoli B. Eye complaints in the office environment: precorneal tear film integrity influenced by eye blinking efficiency. Occup Environ Med. 2005;62:4-12.

9. Olson MC, Korb DR, Greiner JV. Increase in tear film lipid layer thickness following treatment with warm compresses in patients with meibomian gland dysfunction. Eye Contact Lens. 2003;29:96-9.

10. Craig JP, Tomlinson A. Importance of the lipid layer in human tear film stability and evaporation. Optom Vis Sci. 1997;74:8-13.

11. Thode AR, Latkany RA. Current and emerging therapeutic strategies for the treatment of Meibomian Gland Dysfunction (MGD). Drugs. 2015;75:1177-85.

12. Toyos R, McGill W, Briscoe D. Intense pulsed light treatment for dry eye disease due to meibomian gland dysfunction; a 3-year retrospective study. Photomed Laser Surg. 2015:33:41-6.

13. Matsumoto $Y$, Dogru M, Goto E, Ishida R, Kojima T, Onguchi T, et al. Efficacy of a new warm moist air device on tear functions of patients with simple meibomian gland dysfunction. Cornea. 2006;25:644-50.

14. Paramdeep Singh B, Shehzad Anjam N, James SW. Randomised masked clinical trial of the MGDRx EyeBag for the treatment of meibomian gland dysfunction-related evaporative dry eye. Br J Ophthalmol. 2014;98:1707-11.

15. Korb DR, Blackie CA. Using goggles to increase periocular humidity and reduce dry eye symptoms. Eye Contact Lens. 2013;39:273-6.

16. Pult $\mathrm{H}$, Riede-Pult $\mathrm{BH}$, Purslow C. A comparison of an eyelid-warming device to traditional compress therapy. Optom Vis Sci. 2012;89:E1035-41.

17. Kojima T, Ibrahim OM, Wakamatsu T, Tsuyama A, Ogawa J, Matsumoto Y, et al. The impact of contact lens wear and visual display terminal work on ocular surface and tear functions in office workers. Am J Ophthalmol. 2011; 152:933-40.e2.

18. Schiffman RM, Christianson MD, Jacobsen G, Hirsch JD, Reis BL. Reliability and validity of the ocular surface disease index. Arch Ophthalmol. 2000;118: 615-21.

19. Ozcura F, Aydin S, Helvaci MR. Ocular surface disease index for the diagnosis of dry eye syndrome. Ocul Immunol Inflamm. 2007;15:389-93. 
20. Sack RA, Beaton A, Sathe S, Morris C, Willcox M, Bogart B. Towards a closed eye model of the pre-ocular tear layer. Prog Retin Eye Res. 2000;19:649-68.

21. Miller KL, Walt JD. Minimal clinically important difference for the ocular surface disease index. Arch Ophthalmol. 2010;128:94-101.

22. Amparo F, Schaumberg DA, Dana R. Comparison of Two Questionnaires for Dry Eye Symptom Assessment: The Ocular Surface Disease Index and the Symptom Assessment in Dry Eye. Ophthalmology. 2015;122:1498-503.

23. Wewers ME, Lowe NK. A critical review of visual analogue scales in the measurement of clinical phenomena. Res Nurs Health. 1990;13:227-36.

24. Barr JT, Schechtman KB, Fink BA, Pierce GE, Pensyl CD, Zadnik K, et al. Corneal scarring in the Collaborative Longitudinal Evaluation of Keratoconus (CLEK) study: baseline prevalence and repeatability of detection. Cornea. 1999:18:34-46.

25. Abdelfattah NS, Dastiridou A, Sadda SR, Lee OL. Noninvasive imaging of tear film dynamics in eyes with ocular surface disease. Cornea. 2015;34(Suppl 10):S48-52.

26. Wang X, Lu X, Yang J, Wei R, Yang L, Zhao S, et al. Evaluation of dry eye and meibomian gland dysfunction in teenagers with myopia through noninvasive keratograph. J Ophthalmol. 2016;2016:6761206.

27. Wu SQ, Hong JX, Tian LJ, Cui XH, Sun XH, Xu JJ. Assessment of bulbar redness with a newly developed keratograph. Optom Vis Sci. 2015;92:892-9.

28. Koh S, Ikeda C, Watanabe S, Oie Y, Soma T, Watanabe H, et al. Effect of noninvasive tear stability assessment on tear meniscus height. Acta Ophthalmol. 2015;93:e135-9.

29. Bilkhu PS, Naroo SA, Wolffsohn JS. Randomised masked clinical trial of the MGDRx EyeBag for the treatment of meibomian gland dysfunction-related evaporative dry eye. Br J Ophthalmol. 2014;98:1707-11.

30. Mitra M, Menon GJ, Casini A, Hamada S, Adams D, Ricketts C, et al. Tear film lipid layer thickness and ocular comfort after meibomian therapy via latent heat with a novel device in normal subjects. Eye (London, England). 2005; 19:657-60.

31. Tsubota K, Nakamori K. Dry eyes and video display terminals. N Engl J Med. 1993:328:584.

32. Hirayama M, Murat D, Liu Y, Kojima T, Kawakita T, Tsubota K. Efficacy of a novel moist cool air device in office workers with dry eye disease. Acta Ophthalmol. 2013;91:756-62.

\section{Submit your next manuscript to BioMed Central and we will help you at every step:}

- We accept pre-submission inquiries

- Our selector tool helps you to find the most relevant journal

- We provide round the clock customer support

- Convenient online submission

- Thorough peer review

- Inclusion in PubMed and all major indexing services

- Maximum visibility for your research

Submit your manuscript at www.biomedcentral.com/submit

Biomed Central 